\title{
mmpp: A Package for Calculating Similarity and Distance Metrics for Simple and Marked Temporal Point Processes
}

\author{
by Hideitsu Hino, Ken Takano, and Noboru Murata
}

\begin{abstract}
A simple temporal point process (SPP) is an important class of time series, where the sample realization of the process is solely composed of the times at which events occur. Particular examples of point process data are neuronal spike patterns or spike trains, and a large number of distance and similarity metrics for those data have been proposed. A marked point process (MPP) is an extension of a simple temporal point process, in which a certain vector valued mark is associated with each of the temporal points in the SPP. Analyses of MPPs are of practical importance because instances of MPPs include recordings of natural disasters such as earthquakes and tornadoes. In this paper, we introduce the R package mmpp, which implements a number of distance and similarity metrics for SPPs, and also extends those metrics for dealing with MPPs.
\end{abstract}

\section{Introduction}

A random point process is a mathematical model for describing a series of discrete events (Snyder and Miller, 1991). Let $\mathcal{X}=\left\{t ; t_{0} \leq t \leq t_{0}+T\right\}$ be the base space, on which an event occurs. The base space can be quite abstract, but here we will take $\mathcal{X}$ to be a semi-infinite real line representing time. A set of ordered points on $\mathcal{X}$ is denoted as $x=\left\{x_{1}, x_{2}, \ldots, x_{n}\right\}, x_{i} \leq x_{i+1}$, and called a sample realization, or simply realization of a point process.

Reflecting the importance of the analysis of point processes in a broad range of science and engineering problems, there are already some $\mathrm{R}$ packages for modeling and simulating point processes such as splancs (Rowlingson and Diggle, 1993), spatstat (Baddeley and Turner, 2005), PtProcess (Harte, 2010), and stpp (Gabriel et al., 2013). These packages support various approaches for the analysis of both simple and marked spatial or spatio-temporal point processes, namely, estimating an intensity function for sample points, visualizing the observed sample process, and running simulations based on the specified models.

To complement the above mentioned packages, in mmpp (Hino et al., 2015), we focus on the similarity or distance metrics between realizations of point processes. Similarity and distance metrics are fundamental notions for multivariate analysis, machine learning and pattern recognition. For example, with an appropriate distance metric, a simple $k$ nearest neighbor classifier and regressor (Cover and Hart, 1967) works in a satisfactory way. Also, kernel methods (Shawe-Taylor and Cristianini, 2004) are a well known and widely used framework in machine learning, in which inferences are done solely based on the values of kernel function, which is considered as a similarity metric between two objects.

As for the distance and similarity metric for point processes, vast amount of methods are developed in the field of neuroscience (Kandel et al., 2000). In this field, neural activities are recorded as sequences of spikes (called spike trains), which is nothing but a realization of a simple point process (SPP). By its nature, the responses of neurons to the same stimulus can be different. To claim the repeatability and the reliability of experimental results, a number of different distance and similarity metrics between sequence of spikes are developed (Victor, 2005; Schreiber et al., 2003; Kreuz et al., 2007; Quian Quiroga et al., 2002; van Rossum, 2001).

The package mmpp categorizes commonly used metrics for spike trains and offers implementations for them. Since a spike train is a realization of a simple point process, the original metrics developed in the field of neuroscience do not consider marked point process (MPP) realizations. mmpp extends conventional metrics for SPPs to MPPs. We have two main aims in the development of mmpp:

1. to have a systematic and unified platform for calculating the similarities and distances between SPPs, and

2. to support MPPs to offer a platform for performing metric-based analysis of earthquakes, tornados, epidemics, or stock exchange data. 

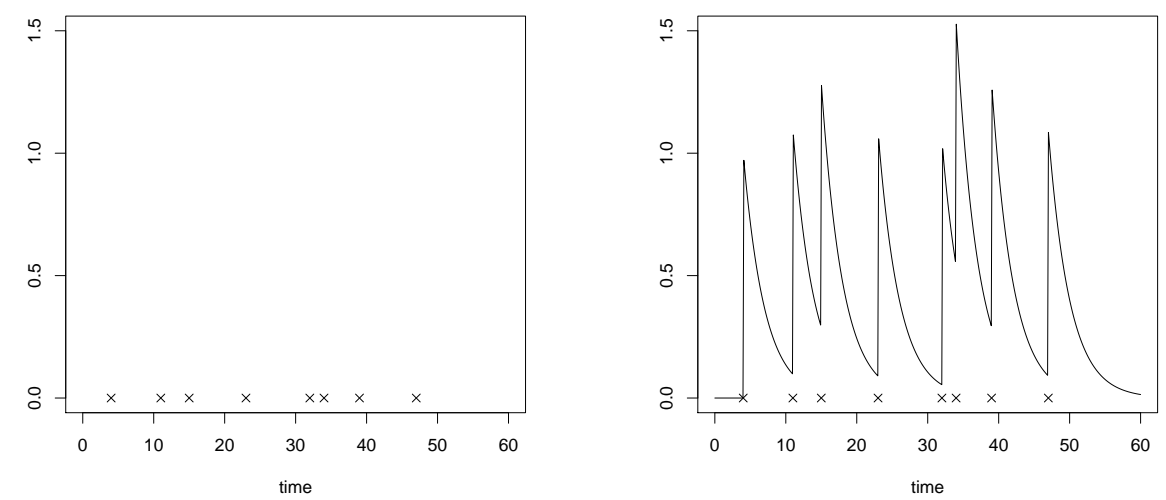

Figure 1: An example of continuation by smoothing. Left: event timings are marked with $\times$. Right: the corresponding continuous function $v_{x}(t)$.

\section{Distances and similarities for point processes}

Since realizations of temporal SPPs are ordered sets of the events, the commonly used Euclidean distance and inner product cannot be directly defined between them. Most of the metrics for SPP realizations first transform the realizations $x=\left\{x_{1}, \ldots, x_{n}\right\}, x_{i} \leq x_{i+1}$ and $y=\left\{y_{1}, \ldots, y_{m}\right\}, ; y_{j} \leq$ $y_{j+1}$ to continuous functions $v_{x}(t)$ and $v_{y}(t)$, then define the distance or similarity metric between them. Based on the transformations, we categorize conventional methods for defining metrics on SPP realizations, and explain one by one in the following subsections.

We note that there are some attempts to directly define distances between SPP realizations. One of the most principled and widely used methods is based on the edit distance (Victor, 2005), and this method is extended to deal with MPP realizations by Suzuki et al. (2010). However, this approach is computationally expensive and prohibitive for computing the distance between spike trains with even a few dozen spikes. We exclude this class of metric from the current version of $\mathbf{m m p p}$.

In the following, we often use kernel smoothers and step functions for transforming SPP realizations. For notational convenience, we denote a kernel smoother with parameter $\tau$ by $h_{\tau}(t)$, and the Heaviside step function by

$$
u(t)= \begin{cases}1, & t \geq 0 \\ 0, & t<0 .\end{cases}
$$

Examples of smoothers include the Gaussian kernel smoother $h_{\tau}^{g}(t)=\exp \left(-t^{2} /\left(2 \tau^{2}\right)\right) / \sqrt{2 \pi \tau^{2}}$ and the Laplacian kernel smoother $h_{\tau}^{l}(t)=\exp (-|t| / \tau) /(2 \tau)$.

\section{Filtering to a continuous function}

The most commonly used and intensively studied metrics for spike trains are based on the mapping of an event sequence to a real valued continuous function as

$$
x=\left\{x_{1}, \ldots, x_{n}\right\} \Rightarrow v_{x}(t)=\frac{1}{n} \sum_{i=1}^{n} h_{\tau}\left(t-x_{i}\right) \cdot u\left(t-x_{i}\right) .
$$

Figure 1 illustrates the transformation of an event sequence to a continuous function by the smoothing method.

Then, the inner product of $x$ and $y$ is defined by the usual $\ell_{2}$ inner product in functional space by

$$
k(x, y)=\int_{0}^{\infty} \mathrm{d} t v_{x}(t) v_{y}(t) \in[-\infty, \infty],
$$

and similarly the distance is defined by

$$
d(x, y)=\sqrt{\int_{0}^{\infty} \mathrm{d} t\left(v_{x}(t)-v_{y}(t)\right)^{2}}
$$

When we use the Laplacian smoother $h_{\tau}^{l}(t)=\exp (-|t| / \tau) /(2 \tau)$, the similarity and distance are 
analytically given as

$$
k(x, y)=\int_{0}^{\infty} \mathrm{d} t v_{x}(t) v_{y}(t)=\frac{1}{4 \tau n m} \sum_{i=1}^{n} \sum_{j=1}^{m} \exp \left(-\frac{1}{\tau}\left|x_{i}-y_{j}\right|\right),
$$

and

$$
\begin{aligned}
d^{2}(x, y)= & k(x, x)+k(y, y)-2 k(x, y) \\
= & \frac{1}{4 \tau n^{2}} \sum_{i=1}^{n} \sum_{j=1}^{n} \exp \left(-\frac{\left|x_{i}-x_{j}\right|}{\tau}\right)+\frac{1}{4 \tau m^{2}} \sum_{i=1}^{m} \sum_{j=1}^{m} \exp \left(-\frac{\left|y_{i}-y_{j}\right|}{\tau}\right) \\
& -\frac{1}{2 \tau n m} \sum_{i=1}^{n} \sum_{j=1}^{m} \exp \left(-\frac{\left|x_{i}-y_{j}\right|}{\tau}\right)
\end{aligned}
$$

respectively. This distance eq. (6) is adopted by van Rossum (2001) for measuring the distance between spike trains. On the other hand, Schreiber et al. (2003) proposed to use the correlation defined by

$$
\operatorname{cor}(x, y)=\frac{\int_{0}^{\infty} \mathrm{d} t v_{x}(t) v_{y}(t)}{\sqrt{\int_{0}^{\infty} \mathrm{d} t v_{x}(t) v_{x}(t)} \sqrt{\int_{0}^{\infty} \mathrm{d} t v_{y}(t) v_{y}(t)}}
$$

to measure the similarity between spike trains. This class of measures is extended to take into account the effect of burst, i.e., the short interval in which events occur in high frequency, and refractory period, i.e., the short interval in which events tend to be suppressed immediately after the previous events (Houghton, 2009; Lyttle and Fellous, 2011). These two effects, namely burst and refractory period, are commonly observed in neural activities. They are also observed in earthquake catalogues. After a large main shock, usually we observe high frequent aftershocks. On the other hand, suppression of events is sometimes happening, possibly because after a big event, the coda is so large that one cannot detect smaller events under the large ongoing signal from the big event (Kagan, 2004; Iwata, 2008).

The filtering-based metric is computed by using the function fmetric in mmpp. The first two arguments S1 and S2 are the (marked) point process realizations in the form of a 'matrix' object. The first column of S1 and S2 are the event timings and the rest are the marks. The argument measure can be either "sim" or "dist", indicating similarity or distance, respectively. By default, the function assumes the Laplacian smoother. When the argument $h$ of function fmetric is set to a function with scaling parameter $\tau$ as

$>$ fmetric(S1, S2, measure $=$ "sim", h $=$ function $\left.(x, \operatorname{tau}) \exp \left(-\mathrm{x}^{\wedge} 2 / \operatorname{tau}\right), \operatorname{tau}=1\right)$

the integrals in eq. (3) and eq. (4) are numerically done using the R function integrate. The function $h$ should be square integrable and non-negative.

\section{Intensity inner products}

For analysis of point processes, the intensity function plays a central roll. Paiva et al. (2009) proposed to use the intensity function for defining the inner product between SPP realizations. Let $N(t)$ be the number of events observed in the interval $(0, t]$. The intensity function of a counting process $N(t)$ is defined by

$$
\lambda_{x}(t)=\lim _{\epsilon \rightarrow 0} \frac{1}{\epsilon} \operatorname{Pr}[N(t+\epsilon)-N(t)=1] .
$$

We note that we can also consider the conditional intensity function reflecting the history up to the current time $t$, but we only explain the simplest case in this paper. Assuming that the SPP to be analysed is well approximated by a Poisson process, the intensity function is estimated by using a smoother $h_{\tau}(t)$ as

$$
\hat{\lambda}_{x}(t)=\frac{1}{n} \sum_{i=1}^{n} h_{\tau}\left(t-x_{i}\right)
$$

in non-parametric manner (Reiss, 1993). Using the estimates of intensity functions for processes behind $x$ and $y$, Paiva et al. (2009) defined a similarity metric by

$$
k(x, y)=\int_{-\infty}^{\infty} \mathrm{d} t \hat{\lambda}_{x}(t) \hat{\lambda}_{y}(t)=\frac{1}{4 \tau^{2} n m} \sum_{i=1}^{n} \sum_{j=1}^{m} \int_{-\infty}^{\infty} \mathrm{d} t h_{\tau}\left(t-x_{i}\right) h_{\tau}\left(t-y_{j}\right) .
$$

Particularly, when we use a Gaussian smoother $h_{\tau}^{g}(t)=\exp \left(-t^{2} /\left(2 \tau^{2}\right)\right) / \sqrt{2 \pi \tau}$, the integral is 
analytically computed and we obtain an explicit formula

$$
k(x, y)=\int_{-\infty}^{\infty} \mathrm{d} t \hat{\lambda}_{x}(t) \hat{\lambda}_{y}(t)=\frac{1}{4 \sqrt{\pi} \tau n m} \sum_{i=1}^{n} \sum_{j=1}^{m} \exp \left(-\frac{\left(x_{i}-y_{j}\right)^{2}}{4 \tau^{2}}\right) .
$$

The distance metric is also defined as

$$
d(x, y)=\int_{-\infty}^{\infty} \mathrm{d} t\left(\hat{\lambda}_{x}(t)-\hat{\lambda}_{y}(t)\right)^{2},
$$

and it is also simplified when we use the Gaussian smoother.

This class of measures is most in alignment with the statistical model of point processes. We estimated the intensity function in a versatile non-parametric approach, but it is reasonable to use other models such as the Hawkes model (Hawkes, 1971) when we should include the self-exciting nature of the process.

The intensity inner product metric is computed by using the function iipmetric in mmpp. In the current version, the function assumes the Gaussian smoother, and its scaling parameter is specified by the argument tau as

$>$ iipmetric(S1, S2, measure = "sim", tau $=1)$

\section{Co-occurrence metric}

For comparing two SPP realizations, it is natural to count the number of events which can be considered to be co-occurring. There are two metrics for SPP realizations based on the notion of co-occurrence.

The first one proposed by Quian Quiroga et al. (2002) directly counts near-by events. The closeness of two events are defined by adaptively computed thresholds, making the method free from tuning parameters. Suppose we have two SPP realizations $x=\left\{x_{1}, \ldots, x_{n}\right\}$ and $y=\left\{y_{1}, \ldots, y_{m}\right\}$. For any events $x_{i} \in x$ and $y_{j} \in y$, a threshold under which the two events are considered to be synchronous with each other is defined as half of the minimum of the four inter event intervals around these two events:

$$
\tau_{i j}=\frac{1}{2} \min \left\{x_{i+1}-x_{i}, x_{i}-x_{i-1}, y_{j+1}-y_{j}, y_{j}-y_{j-1}\right\} .
$$

We note that $\tau_{i j}$ in the above definition depends on $x$ and $y$, though, for the sake of notational simplicity, we simply denote it by $\tau_{i j}$. Then, the function that counts the number of events in $y$ which is coincided with those in $x$ is defined by

$$
\begin{aligned}
c(x \mid y) & =\sum_{i=1}^{n} \sum_{j=1}^{m} P_{i j}, \\
P_{i j} & =\left\{\begin{array}{lc}
1, & 0<x_{i}-y_{j}<\tau_{i j}, \\
1 / 2, & x_{i}=y_{j}, \\
0, & \text { otherwise. }
\end{array}\right.
\end{aligned}
$$

Using this counting function, a similarity measure between $x$ and $y$ is defined as

$$
k(x, y)=\frac{c(x \mid y)+c(y \mid x)}{\sqrt{n m}},
$$

and a distance measure is obtained by the transformation (Lyttle and Fellous, 2011):

$$
d(x, y)=1-k(x, y) .
$$

The second metric based on the counting co-occurrence is proposed by Hunter and Milton (2003), which transforms $x$ to a continuous function $v_{x}(t)$, and sums up the near-by events in proportion to their degree of closeness. Denoting the closest event time in $y$ from an event $x_{i} \in x$ by $y_{\left(x_{i}\right)}$, we define a function which measures degree of closeness by

$$
d c_{\tau}\left(x_{i}\right)=\exp \left(-\frac{\left|x_{i}-y_{\left(x_{i}\right)}\right|}{\tau}\right) .
$$

Then, a similarity metric between $x$ and $y$ is defined by

$$
k(x, y)=\frac{\frac{1}{n} \sum_{i=1}^{n} d c_{\tau}\left(x_{i}\right)+\frac{1}{m} \sum_{j=1}^{m} d c_{\tau}\left(y_{j}\right)}{2},
$$


and the distance is naturally defined by

$$
d(x, y)=1-k(x, y)
$$

The co-occurrence based metrics are computed by using the function coocmetric. By default, the function assumes the counting similarity in eq. (16). The smoothed counting similarity is computed by specifying the argument type = "smooth" as

$>$ coocmetric(S1, S2, measure = "sim", type = "smooth", tau = 1)

\section{Inter event interval}

Assume an SPP realization $x=\left\{x_{1}, \ldots, x_{n}\right\}, x_{n}<T$ such that for every event time $x_{i}, 0<x_{i}<T$, where $T$ is the horizon of the time interval. In the inter event interval proposed by Kreuz et al. (2007), the SPP realization $x$ is first modified to include artificial events corresponding to the beginning and end of the interval as

$$
x=\left\{x_{0}=0, x_{1}, \ldots, x_{n}, x_{n+1}=T\right\} .
$$

Then each event is mapped to a function $v_{x}(t)$ as

$$
v_{x}(t)=\sum_{i=0}^{n+1} f_{i}(t), \quad f_{i}(t)= \begin{cases}0, & t \notin\left[x_{i}, x_{i+1}\right), \\ x_{i+1}-x_{i}, & t \in\left[x_{i}, x_{i+1}\right) .\end{cases}
$$

Two SPP realizations $x$ and $y$ are transformed to $v_{x}(t)$ and $v_{y}(t)$, then they are used to define an intermediate function

$$
I_{x y}(t)=\frac{\min \left\{v_{x}(t), v_{y}(t)\right\}}{\max \left\{v_{x}(t), v_{y}(t)\right\}} .
$$

This function takes value 1 when $x$ is identical to $y$, and takes a smaller value when $x$ and $y$ are highly dissimilar. By using this intermediate function, the similarity measure is defined by

$$
k(x, y)=\frac{1}{T} \int_{0}^{T} \mathrm{~d} t I_{x y}(t),
$$

and the distance is defined by

$$
d(x, y)=\frac{1}{T} \int_{0}^{T} \mathrm{~d} t\left(1-I_{x y}(t)\right),
$$

which is originally defined in Kreuz et al. (2007). A simple example of transformation $x \Rightarrow v_{x}(t), y \Rightarrow$ $v_{y}(t)$ and $x, y \Rightarrow 1-I_{x y}(t)$ is illustrated in Figure 2 .

The inter event interval metrics are computed by using the function ieimetric as

$>$ ieimetric(S1, S2, measure = "sim")

\section{Extension to marked point processes}

Sometimes events considered in point processes entail certain vector valued marks. For example, seismic events are characterized by the time point the earthquake happens, and a set of attributes such as magnitude, depth, longitude, and latitude of the hypo-center. To deal with marked point processes, we extend the base space $\mathcal{X}$ to $\mathcal{X}=\left\{t ; t_{0} \leq t \leq t_{0}+T\right\} \times \mathbb{R}^{p}$, the Cartesian product of the time interval $\left[t_{0}, t_{0}+T\right]$ and a region of the $p$ dimensional Euclidean space corresponding to marks. Realizations of MPPs are denoted by, for example, $x=\left\{\left(x_{1}, \boldsymbol{r}_{1}\right), \ldots,\left(x_{n}, \boldsymbol{r}_{n}\right)\right\}$ and $y=\left\{\left(y_{1}, \boldsymbol{s}_{1}\right), \ldots,\left(y_{m}, \boldsymbol{s}_{m}\right)\right\}$.

There might be many possible ways of extension. The packages mmpp takes the simplest way to deal with marks in a unified and computationally efficient manner, namely, the density or weight of marks are included in the metrics for SPPs by Gaussian windowing as shown in eq. (27).

\section{Filtering to continuous function}

In the same manner as eq. (2), the marked point process realization $x=\left\{\left(x_{1}, \boldsymbol{r}_{1}\right), \ldots,\left(x_{n}, \boldsymbol{r}_{n}\right)\right\}$ is transformed to a continuous function as

$$
x=\left\{\left(x_{1}, \boldsymbol{r}_{i}\right), \ldots,\left(x_{n}, \boldsymbol{r}_{n}\right)\right\} \Rightarrow v_{x}(t, z)=\frac{1}{n} \sum_{i=1}^{n} h_{M}\left(\boldsymbol{z}-\boldsymbol{r}_{i}\right) h_{\tau}\left(t-x_{i}\right) \cdot u\left(t-x_{i}\right),
$$




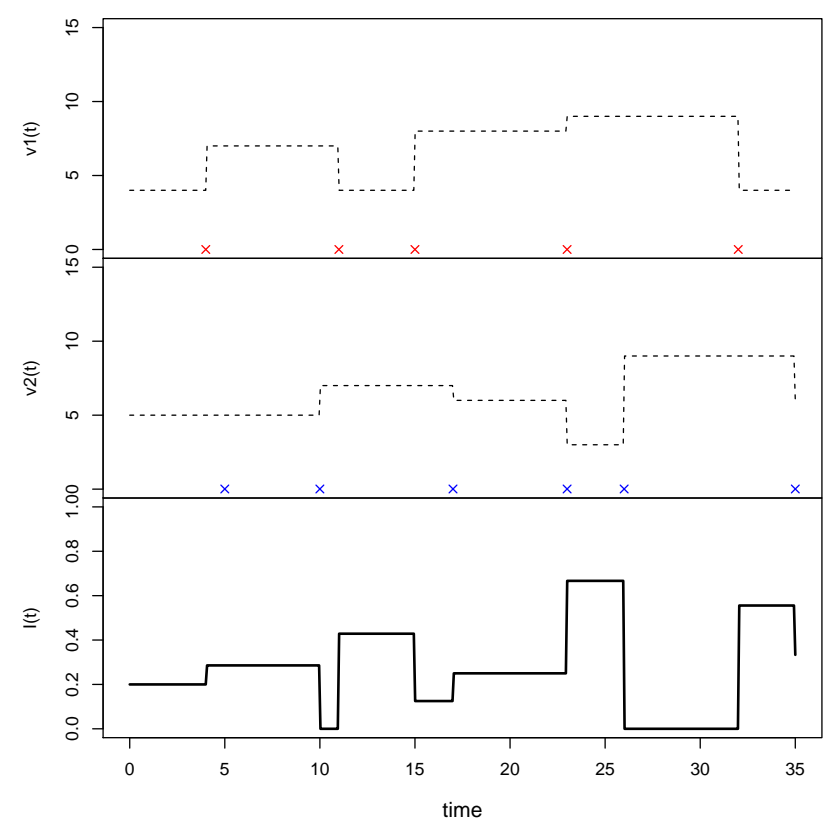

Figure 2: Example of the transformation of two point process realizations $x$ and $y$ into the intermediate function $I_{x y}$. The top panel shows $x$ and corresponding continuous function $v_{x}(t)$. The middle panel shows $y$ and $v_{y}(t)$. The bottom panel shows the intermediate function $1-I_{x y}(t)$.

where

$$
h_{M}(z)=(2 \pi)^{-p / 2}|M|^{1 / 2} \exp \left(-\frac{1}{2} z^{\top} M z\right)=(2 \pi)^{-p / 2}|M|^{1 / 2} \exp \left(-\frac{1}{2}\|z\|_{M}^{2}\right), M \in \mathbb{R}^{p \times p},
$$

where $|M|$ is the determinant of a matrix $M$, and $\|z\|_{M}^{2}=z^{\top} M z$. Integrating with respect to both time $t$ and mark $z$, we define the inner product by

$$
k(x, y)=\int_{\mathbb{R}^{p}} \mathrm{~d} z \int_{0}^{\infty} \mathrm{d} t v_{x}(t, z) v_{y}(t, z),
$$

and the distance by

$$
d^{2}(x, y)=\int_{\mathbb{R}^{p}} \mathrm{~d} z \int_{0}^{\infty} \mathrm{d} t\left(v_{x}(t, z)-v_{y}(t, z)\right)^{2} .
$$

By virtue of Gaussian windowing, the integral with respect to the mark is explicitly written as

$$
\int_{\mathbb{R}^{p}} \mathrm{~d} z \exp \left(-\frac{1}{2}\left\|z-\boldsymbol{r}_{i}\right\|_{M}^{2}-\frac{1}{2}\left\|z-s_{j}\right\|_{M}^{2}\right)=(2 \pi)^{\frac{p}{2}} \sqrt{|2 M|} \exp \left(-\frac{1}{4}\left\|\boldsymbol{r}_{i}-\boldsymbol{s}_{j}\right\|_{M}^{2}\right) .
$$

Furthermore, when we use Laplacian smoother for transforming temporal SPPs, we obtain

$$
k(x, y)=\frac{|M|^{1 / 2}}{2^{p+2} \pi^{p / 2} \tau n m} \sum_{i=1}^{n} \sum_{j=1}^{m} \exp \left(-\frac{\left\|\boldsymbol{r}_{i}-\boldsymbol{s}_{j}\right\|_{M}^{2}}{4}\right) \exp \left(-\frac{\left|x_{i}-y_{j}\right|}{\tau}\right) .
$$

The distance metric is also calculated in the same manner.

We note that the effect of marks depend on the units used for the various marks. It is reasonable to estimate the variance of each mark, and set the diagonal elements of $M$ to be reciprocal of the variances, which is adopted as the default setting for $M$ in mmpp.

\section{Intensity inner product}

Extending kernel estimation eq. (9) to multivariate kernel estimation as

$$
\hat{\lambda}_{x}(t, z)=\frac{1}{n} \sum_{i=1}^{n} h_{M}\left(z-r_{i}\right) h_{\tau}\left(t-x_{i}\right),
$$

we obtain the estimate of the intensity function of the marked point process. We note that kernel density estimation for multidimensional variables is inaccurate in general, and we can instead estimate 
the ground intensity function $\lambda(t)$ and the density function for mark $\lambda(z)$ separately. However, in many applications, the dimension of marks is not so high, and currently we adopt the kernel based estimator in eq. (32). The intensity inner product for MPP realizations is then defined by

$$
k(x, y)=\int_{\mathbb{R}^{p}} \mathrm{~d} z \int_{-\infty}^{\infty} \mathrm{d} t \hat{\lambda}_{x}(t, z) \hat{\lambda}_{y}(t, z) .
$$

When we use the Gaussian smoother $h_{\tau}^{g}(t)=\exp \left(-t^{2} / \tau\right) / \sqrt{2 \pi \tau^{2}}$, the integral is explicitly computed and we obtain

$$
k(x, y)=\frac{1}{\pi^{(p+1) / 2} \tau^{1 / 2}|M|^{1 / 2} n m} \sum_{i=1}^{n} \sum_{j=1}^{m} \exp \left(-\frac{\left(x_{i}-y_{j}\right)^{2}}{2 \tau}\right) \exp \left(-\frac{\left\|\boldsymbol{r}_{i}-\boldsymbol{s}_{j}\right\|_{M}^{2}}{4}\right) .
$$

The distance metric is also defined by

$$
d(x, y)=\int_{\mathbb{R}^{p}} \mathrm{~d} z \int_{-\infty}^{\infty} \mathrm{d} t\left(\hat{\lambda}_{x}(t, z)-\hat{\lambda}_{y}(t, z)\right)^{2} .
$$

For estimating the intensity function, a simple Poisson process is assumed. This assumption is relaxed with more flexible models such as the ETAS model (Ogata, 1988, 1998), where the intensity function is estimated using the R packages SAPP (of Statistical Mathematics, 2014) and etasFLP (Chiodi and Adelfio, 2015), for example. The extension of the intensity-based metric to support other forms of intensity estimation such as the Hawkes and ETAS models remains our important future work.

\section{Co-occurrence metric}

To extend the co-occurrence metric based on counting the synchronous events, eq. (15) is replaced with a weighted counter

$$
P_{i j}=\exp \left(-\left\|\boldsymbol{r}_{i}-\boldsymbol{s}_{j}\right\|_{M}^{2}\right) \times\left\{\begin{array}{lc}
1, & 0<x_{i}-y_{j}<\tau_{i j} \\
1 / 2, & x_{i}=y_{j}, \\
0, & \text { otherwise }
\end{array}\right.
$$

To extend the co-occurrence metric based on the smoothed count of the synchronous events, eq. (18) is replaced with a weighted smoothed counter

$$
d c_{\tau, M}\left(x_{i}\right)=\exp \left(-\left\|\boldsymbol{r}_{i}-\boldsymbol{s}_{\left(x_{i}\right)}\right\|_{M}^{2}\right) \times \exp \left(-\frac{\left|x_{i}-y_{\left(x_{i}\right)}\right|}{\tau}\right) .
$$

\section{Inter event interval}

To weight the inter event interval by using marks associated with two MPP realizations $x$ and $y$, we define index extraction operators as follows. We modify an MPP realization $x=\left\{\left(x_{1}, \boldsymbol{r}_{1}\right), \ldots,\left(x_{n}, \boldsymbol{r}_{n}\right)\right\}$ to include artificial events and marks corresponding to the beginning and end of the interval as

$$
x=\left\{\left(x_{0}=0, \boldsymbol{r}_{0}=\mathbf{0}\right),\left(x_{1}, \boldsymbol{r}_{1}\right), \ldots,\left(x_{n}, \boldsymbol{r}_{n}\right),\left(x_{n+1}=T, \boldsymbol{r}_{n+1}=\mathbf{0}\right)\right\} .
$$

Then we define operators

$$
\begin{aligned}
\underline{q}:[0, T] \times \mathcal{X} & \rightarrow \mathbb{R} \\
(t, x) & \mapsto i, \quad \text { s.t. } \quad t \in\left[x_{i}, x_{i+1}\right], \\
\bar{q}:[0, T] \times \mathcal{X} & \rightarrow \mathbb{R} \\
(t, x) & \mapsto i+1, \quad \text { s.t. } \quad t \in\left[x_{i}, x_{i+1}\right] .
\end{aligned}
$$

The intermediate function eq.(23) is modified to take into account the dissimilarity of marks:

$$
I_{x y}(t)=\frac{\min \left(v_{x}(t), v_{y}(t)\right)}{\max \left(v_{x}(t), v_{y}(t)\right)} \frac{\exp \left(-\left\|\boldsymbol{r}_{q(t, x)}-\boldsymbol{s}_{\underline{q}(t, y)}\right\|_{M}^{2}\right)+\exp \left(-\left\|\boldsymbol{r}_{\bar{q}(t, x)}-s_{\bar{q}(t, y)}\right\|_{M}^{2}\right)}{2} .
$$

The distance and similarity are then calculated using eq. (25) and eq. (24).

The usage of the functions fmetric, iipmetric, coocmetric, and ieimetric does not change for marked point process data, except for one additional argument $\mathrm{M}$, which is the precision matrix $M$ in 


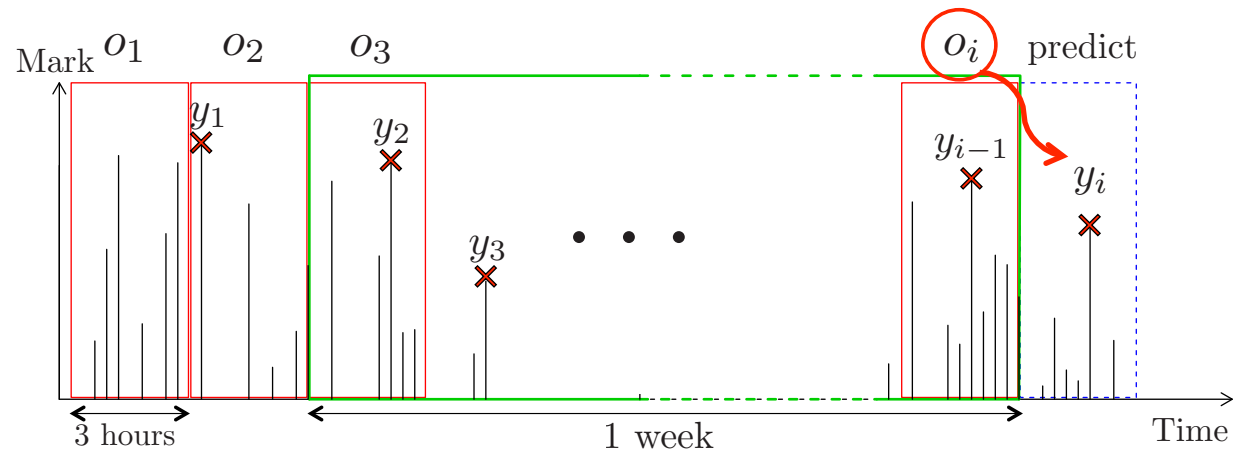

Figure 3: An illustrative diagram of the problem setting. The horizontal axis corresponds to time, and the vertical axis shows marks. Though the dimension of the mark is four, it is shown as onedimensional axis. The process is split by a three hour window, and each window is assigned an output variable, which is the maximum magnitude in the next time window. Using the past one week data, the output variable of the next time window is predicted by nearest neighbor regression.

eq. (27). By default, it is automatically set to the diagonal matrix with the diagonal elements equal to the reciprocal of the variance of corresponding marks of S1 and S2. We can also specify the matrix $M$ manually as

$>$ fmetric(S1, S2, measure = "sim", M = diag(3))

where the number of marks is assumed to be three.

\section{An example with the Miyagi20030626 data set}

This section illustrates the use of the package with a simple experiment. We use the Miyagi20030626 dataset contained within the package.

$$
\begin{aligned}
& >\text { library (mmpp) } \\
& >\text { data(Miyagi20030626) }
\end{aligned}
$$

The dataset is composed of 2305 aftershocks of the 26th July 2003 earthquake of M6.2 at the northern Miyagi-Ken Japan, which is a reparameterization of the main2006 JUL26 dataset from the SAPP package. Each record has 5 dimensions, time, longitude, latitude, depth, and magnitude of its hypo-center. The time is recorded in seconds from the main shock.

To illustrate the use of the package, we consider a simple prediction task. We first split the original dataset by a time-window of length $60 \times 60 \times 3$, which means that the time interval of each partial point process split by this window is three hours.

$>$ sMiyagi <- splitMPP(Miyagi20030626, $\mathrm{h}=60 * 60 * 3$, scaleMarks $=$ TRUE) $\$ S$

Then, the maximum magnitude in each partial point process realization is computed.

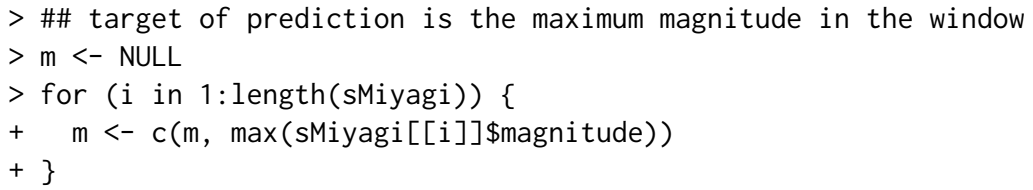

The task we consider is the prediction of the maximum magnitude in the next three hours using the past one week of data. We formulate this problem as a regression problem. Let the partial point process realization in the $i$-th window be $o_{i}$, and let the maximum magnitude in the $i+1$-th window be $m_{i}$. Then the problem is predicting $m_{i+1}$ given $o_{i+1}$ and the past $24 \times 7 / 3=56$ hours of data $\left\{\left(o_{i-\ell}, m_{i-\ell}\right)\right\}_{\ell=0}^{55}$. See Figure 3 for an illustrative diagram of the problem setting.

$>\mathrm{m}<-\mathrm{m}[-1]$

$>$ sMiyagi[[length(sMiyagi)]]<- NULL

$>$ \#\# number of whole partial MPPs split by a 3-hour time window

$>\mathrm{N}<-$ length(sMiyagi)

$>$ \#\# training samples are past one week data

$>\mathrm{Ntr}<-24 * 7 / 3$

$>$ \#\# number of different prediction methods

$>\mathrm{Nd}<-10$ 
For the purpose of illustrating the use of the package and to show the effect of different similarity metrics, we adopt the nearest neighbor regression. That is, given the current realization $o_{i}$, we find the most similar realization $o_{j} \in\left\{o_{i-l}\right\}_{l=0}^{55}$, and use the corresponding maximum magnitude $m_{j}$ as the predictor for $m_{i+1}$. We use ten different similarity metrics supported in the package, and evaluate the mean absolute errors. The metrics used for these experiments are the filter based metric in eq. (3), the intensity inner product metric in eq. (10), the co-occurrence with counting in eq. (16), the co-occurrence with smoothed counting in eq. (19), and the inter event interval metric in eq. (24), and their MPP extensions.

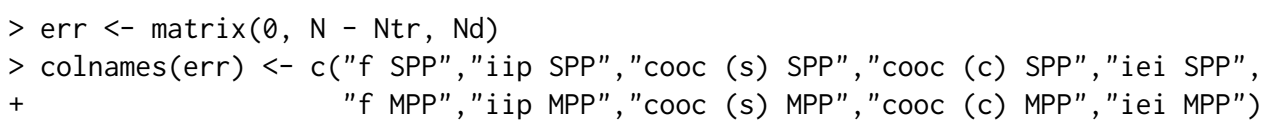

The following code performs the above explained experiment.

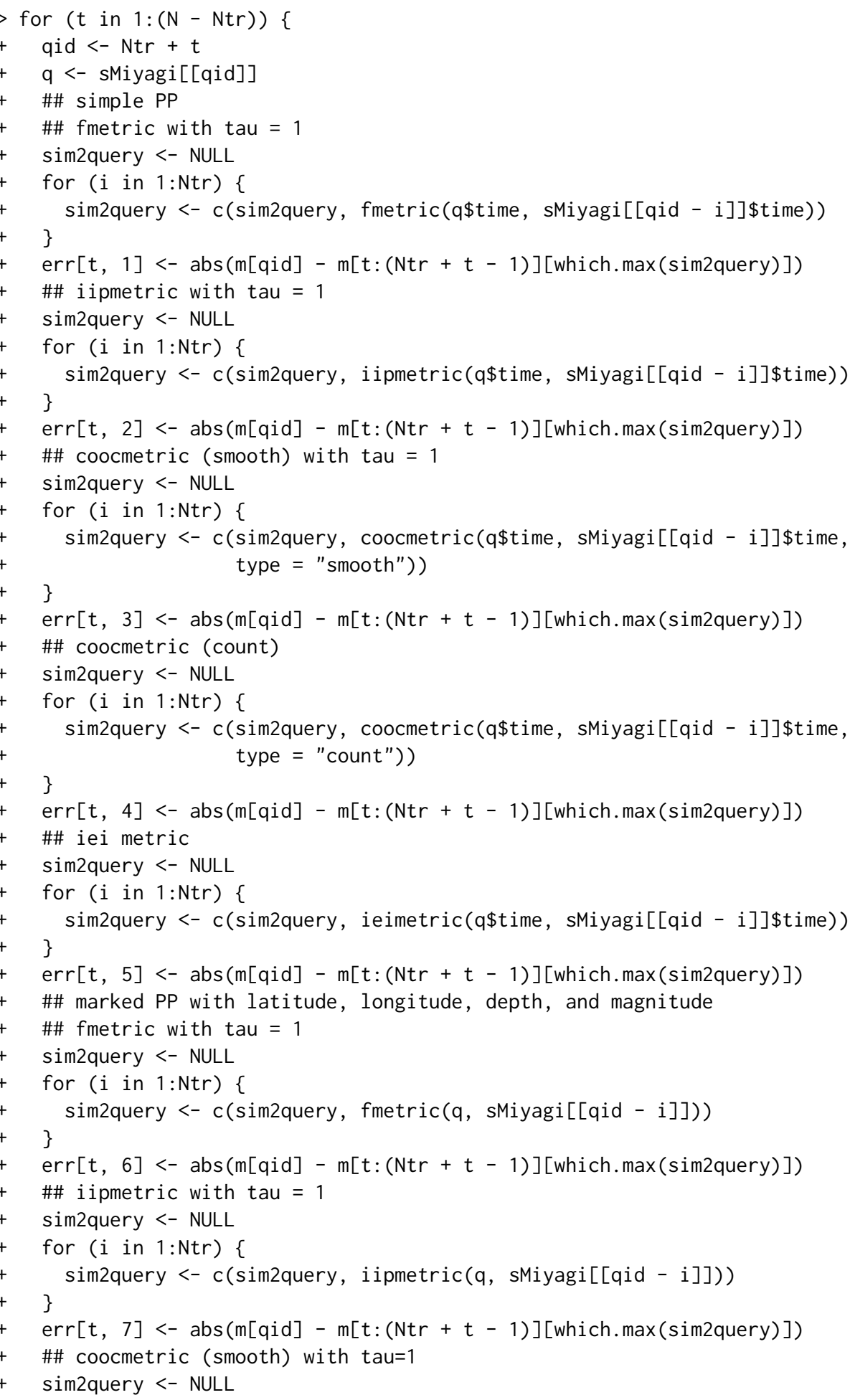




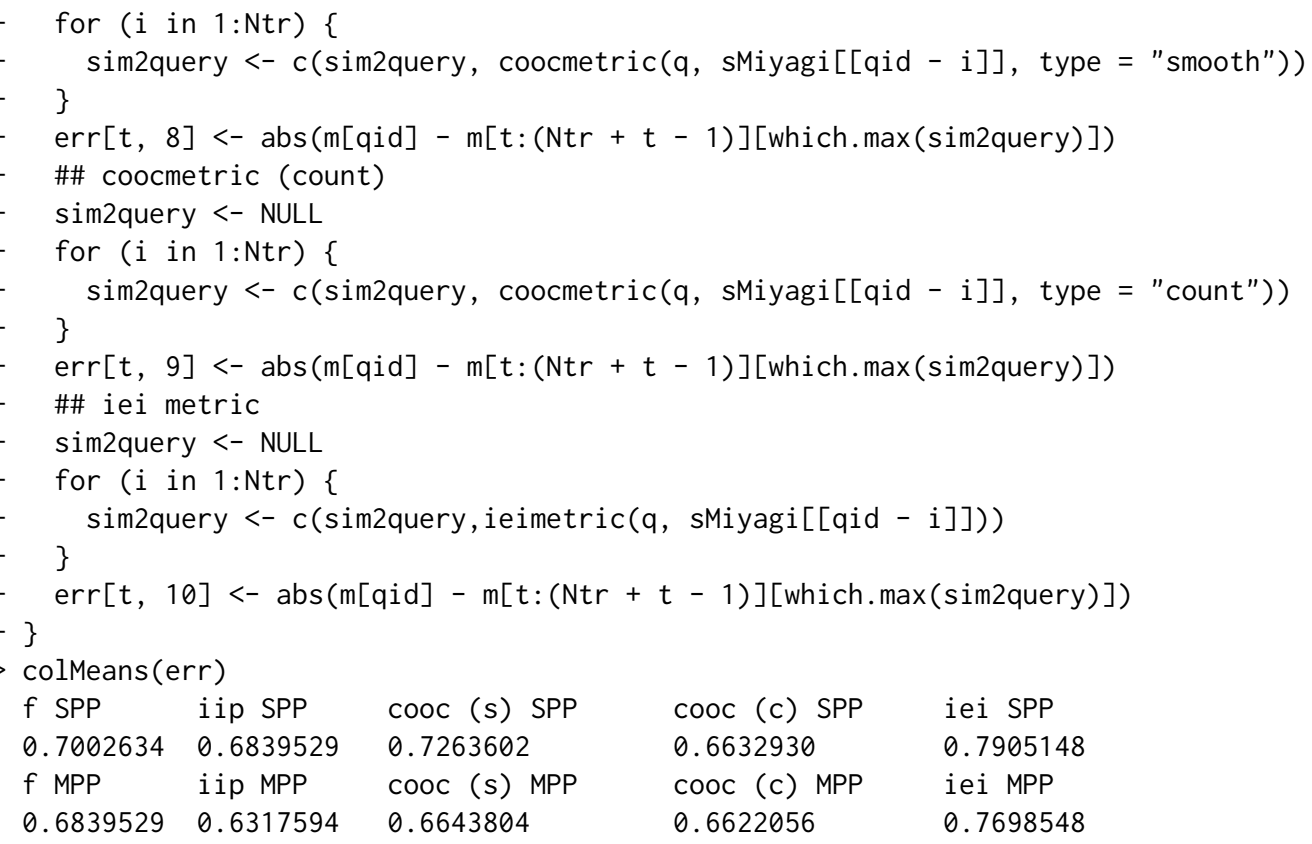

From this simple example, we can see that the prediction accuracy is improved by taking the marks into account.

\section{Summary and future directions}

mmpp is the first $\mathrm{R}$ package dedicated to the calculation of the similarity and distance metrics for marked point process realizations. It provides the implementation of several similarity metrics for simple point processes, originally proposed in the literature of neuroscience, and also provides extensions of these metrics to those for marked point processes.

A simple example of a real dataset presented in this paper illustrates the importance of taking the marks into account in addition to the event timings, and it also illustrates the possibilities of the package mmpp with a user guide for practitioners.

The development of the mmpp package has only just begun. Currently, we are considering supporting burst sensitive and refractory period sensitive versions of fmetric, since these properties are commonly observed in both neural activities and seismic event recordings. In the current version of mmpp, for treating MPPs, event timings and marks are assumed to be separable, and all the marks are simultaneously estimated by a kernel density estimator. This is a strong assumption and other possibilities for modeling MPPs should be considered. For example, it is popular to group spatio-temporal events, i.e., event timings and locations, and treat marks such as magnitude in seismic events as purely marks. Then, the separability between marks and spatio-temporal events can be tested by using test statistics proposed in Schoenberg (2004) and Chang and Schoenberg (2011). The separability assumption offers computational advantages, though, it would miss the intrinsic structure and relationship between event timings and marks. In principle, the separability hypothesis should be tested before calculating the metrics. Frameworks for flexible modeling of marked sample sequences with statistical validation such as nonparametric tests would be implemented in future version of mmpp. We are also considering to extend the intensity inner product metric to support other form of intensity estimation such as Hawkes and ETAS models.

Different similarity metrics capture different aspects of the point process realizations. Our final goal of the development of the package $\mathbf{m m p p}$ is to provide a systematic way to select or combine appropriate metrics for analysing given point process realizations and certain tasks such as prediction of magnitude or clustering similar seismic events.

\section{Acknowledgement}

The authors are grateful to T. Iwata for helpful discussions and suggestions. The authors would like to express their special thanks to the editor, the associate editor and three anonymous reviewers whose comments led to valuable improvements of the manuscript. Part of this work is supported by JSPS KAKENHI Grant Number 25870811, 26120504, and 25120009. 


\section{Bibliography}

A. Baddeley and R. Turner. spatstat: An R package for analyzing spatial point patterns. Journal of Statistical Software, 12(6):1-42, 1 2005. URL http://www. jstatsoft.org/v12/i06. [p237]

C.-H. Chang and F. Schoenberg. Testing separability in marked multidimensional point processes with covariates. The Annals of the Institute of Statistical Mathematics, 63(6):1103-1122, 2011. doi: 10.1007/s10463-010-0284-7. [p246]

M. Chiodi and G. Adelfio. etasFLP: Mixed FLP and ML Estimation of ETAS Space-Time Point Processes, 2015. URL https://CRAN.R-project.org/package=etasFLP. R package version 1.3.0. [p243]

T. Cover and P. Hart. Nearest neighbor pattern classification. IEEE Transactions on Information Theory, 13(1):21-27, 1967. doi: 10.1109/TIT.1967.1053964. [p237]

E. Gabriel, B. S. Rowlingson, and P. J. Diggle. stpp: An R package for plotting, simulating and analyzing spatio-temporal point patterns. Journal of Statistical Software, 53(2):1-29, 4 2013. URL http://www. jstatsoft.org/v53/i02. [p237]

D. Harte. PtProcess: An R package for modelling marked point processes indexed by time. Journal of Statistical Software, 35(8):1-32, 2010. URL http://www. jstatsoft. org/v35/i08/. [p237]

A. G. Hawkes. Spectra of some self-exciting and mutually exciting point processes. Biometrika, 58(1): 83-90, 1971. doi: 10.2307/2334319. [p240]

H. Hino, K. Takano, Y. Yoshikawa, and N. Murata. mmpp: Various similarity and distance metrics for marked point processes, 2015. URL https: //CRAN. R-project. org/package=mmpp. [p237]

C. Houghton. Studying spike trains using a van Rossum metric with a synapse-like filter. Journal of Computational Neuroscience, 26(1):149-155, 2009. doi: 10.1007/s10827-008-0106-6. [p239]

J. D. Hunter and J. G. Milton. Amplitude and frequency dependence of spike timing: Implications for dynamic regulation. Journal of Neurophysiology, 90(1):387-394, July 2003. doi: 10.1152/jn.00074.2003. [p240]

T. Iwata. Low detection capability of global earthquakes after the occurrence of large earthquakes: Investigation of the Harvard CMT catalogue. Geophysical Journal International, 174(3):849-856, 2008. doi: 10.1111/j.1365-246X.2008.03864.x. [p239]

Y. Y. Kagan. Short-term properties of earthquake catalogs and models of earthquake source. Bulletin of the Seismological Society of America, 94(4):1207-1228, 2004. doi: 10.1785/012003098. [p239]

E. R. Kandel, J. H. Schwartz, and T. M. Jessell. Principles of Neural Science. McGraw-Hill Medical, 4th edition, July 2000. [p237]

T. Kreuz, J. S. Haas, A. Morelli, H. D. Abarbanel, and A. Politi. Measuring spike train synchrony. Journal of Neuroscience Methods, 165(1):151-161, 2007. doi: 10.1016/j.jneumeth.2007.05.031. [p237, 241]

D. Lyttle and J.-M. Fellous. A new similarity measure for spike trains: Sensitivity to bursts and periods of inhibition. Journal of Neuroscience Methods, 199(2):296-309, 2011. doi: 10.1016/j.jneumeth.2011.05. 005. [p239, 240]

T. I. of Statistical Mathematics. SAPP: Statistical Analysis of Point Processes, 2014. URL https: //CRAN. Rproject. org/package=SAPP. R package version 1.0.4. [p243]

Y. Ogata. Statistical models for earthquake occurrences and residual analysis for point processes. Journal of the American Statistical Association, 83(401):9-27, Mar. 1988. doi: 10.2307/2288914. [p243]

Y. Ogata. Space-time point-process models for earthquake occurrences. The Annals of the Institute of Statistical Mathematics, 50(2):379-402, June 1998. doi: 10.1023/a:1003403601725. [p243]

A. R. C. Paiva, I. Park, and J. C. Príncipe. A reproducing kernel Hilbert space framework for spike train signal processing. Neural Comput., 21(2):424-449, Feb. 2009. doi: 10.1162/neco.2008.09-07-614. [p239]

R. Quian Quiroga, T. Kreuz, and P. Grassberger. Event synchronization: A simple and fast method to measure synchronicity and time delay patterns. Physical Review E, 66:041904, Oct 2002. doi: 10.1103/PhysRevE.66.041904. [p237, 240] 
R.-D. Reiss. A Course on Point Processes. Springer Series in Statistics. Springer, 1993. [p239]

B. Rowlingson and P. Diggle. Splancs: Spatial point pattern analysis code in S-PLUS. Computers $\mathcal{E}$ Geosciences, 19(5):627-655, 1993. doi: 10.1016/0098-3004(93)90099-Q. [p237]

F. P. Schoenberg. Testing separability in spatial-temporal marked point processes. Biometrics, $60(2)$ : 471-481, 2004. doi: 10.1111/j.0006-341X.2004.00192.x. [p246]

S. Schreiber, J. Fellous, D. Whitmer, P. Tiesinga, and T. Sejnowski. A new correlation-based measure of spike timing reliability. Neurocomputing, 52-54(0):925 - 931, 2003. doi: 10.1016/S0925-2312(02)00838X. Computational Neuroscience: Trends in Research 2003. [p237, 239]

J. Shawe-Taylor and N. Cristianini. Kernel Methods for Pattern Analysis. Cambridge University Press, New York, NY, USA, 2004. [p237]

D. L. Snyder and M. I. Miller. Random Point Processes in Time and Space. Springer-Verlag, New York, NY, USA, 2nd edition, 1991. [p237]

S. Suzuki, Y. Hirata, and K. Aihara. Definition of distance for marked point process data and its application to recurrence plot-based analysis of exhange tick data of foreign currencies. International Journal of Bifurcation and Chaos, 20(11):3699-3708, 2010. doi: 10.1142/S0218127410027970. [p238]

M. C. W. van Rossum. A novel spike distance. Neural Computation, 13(4):751-763, 2001. doi: 10.1162/ 089976601300014321. [p237, 239]

J. D. Victor. Spike train metrics. Current Opinion in Neurobiology, 15:585-592, October 2005. doi: 10.1016/j.conb.2005.08.002. [p237, 238]

Hideitsu Hino

Graduate School of Systems and Information Engineering, University of Tsukuba

1-1-1 Tennoudai, Tsukuba, Ibaraki, 305-8573

Japan

hinohide@cs. tsukuba.ac.jp

Ken Takano

School of Science and Engineering, Waseda University

3-4-1 Ohkubo, Shinjuku-ku, Tokyo 169-8555

Japan

ken.takano@toki.waseda.jp

Noboru Murata

School of Science and Engineering, Waseda University

3-4-1 Ohkubo, Shinjuku-ku, Tokyo, 169-8555

Japan

noboru.murata@eb.waseda.ac.jp 